Case Report

\title{
BILATERAL OSSIFIED STYLOHYOID CHAIN - A CASE STUDY
}

\author{
Shivarama C.H. ${ }^{1}$, Bhat Shivarama ${ }^{2}$, Radhakrishna Shetty K. ${ }^{3}$, Vikram S. ${ }^{4}$, Avadhani R. ${ }^{5}$ \\ ${ }^{1} \mathrm{PG} /$ Tutor, ${ }^{2}$ Associate Professor, ${ }^{5}$ Professor, ${ }^{3}$ Assistant Professor, ${ }^{4}$ Assistant Professor, \\ Department of Anatomy, Yenepoya M edical College, Yenepoya University, M angalore - 575 018, India. \\ Correspondence: \\ Ramakrishna Avadhani, \\ M obile : 98452 53560, E-mail : rkavadhani@ rediffmail.com
}

\begin{abstract}
:
The styloid process is a slender bony projection that arises from the inferior surface of the temporal bone just beneath the external auditory meatus and closely related to the stylomastoid foramen. The normal length of SP in an adult is considered to be 20 to $30 \mathrm{~mm}$ however, it is very variably developed, ranging in length from a few millimetres to a few centimetres.

The styloid process is developed at the cranial end of cartilage in the second visceral or hyoid arch by two centers: a proximal, for the tympanohyal, appearing before birth; the other, for the distal stylohyal, after birth. But sometimes the stylohyoid chain may form, that extends between the temporal and hyoid bones which are divided into 4 sections: tympanohyal, stylohyal, ceratohyal and hypohyal. Cartilage that is embryo logically located at the stylohyoid ligament may undergo calcification of varying degrees, which causes variations. Ossified stylohyal ligament parts may merge or leave gaps in between. The anatomy of styloid process has immense embryological, clinical, surgical importance.
\end{abstract}

Keywords: Styloid process, Stylohyoid chain, Reichert's cartilage, Eagle's syndrome.

\section{Introduction :}

The styloid process (SP), slender, pointed, about $2.5 \mathrm{~cm}$ in length, projects anteroinferiorly from the temporal bone's inferior aspect. Its curvature is somewhat variable. Its proximal part (tympanohyal) is ensheathed by the tympanic plate, especially anterolaterally; to its distal part (stylohyal) are attached muscles and ligaments. The process is covered laterally by the parotid gland; facial nerve crosses its base, the external carotid artery its tip, embedded in the gland. Medially the process is separated from the beginning of the internal jugular vein by the attachment of stylopharyngeus ${ }^{1}$.The tip of the SP usually reaches a point medial to the posterior margin of the mandibular ramus. The SP is, however, very variably developed, ranging in length from a few millimetres to a few centimetres, often approximately straight, but on occasion curved; an anteromedial concavity is more common, a posterior concavity is infrequent ${ }^{1}$. Stylohyoid muscle is attached to SP by a slender tendon to its posterior aspect near the base, styloglossus to the tip and adjacent anterior aspect, stylopharyngeus medially to its base and stylomandibular ligament laterally near its tip. From its apex the stylohyoid ligament descends forward to the hyoid's lesser cornu as a cranial suspension. Behind its base the facial nerve emerges from the stylomastoid foramen crossing lateral to the process in the parotid gland ${ }^{1}$.Normally the SP tapers toward its tip that lies in the pharyngeal wall lateral to the tonsillar fossa ${ }^{2}$. The internal carotid artery, internal jugular vein and cranial nerves $\mathrm{X}, \mathrm{XI}$ and XII lie on its medial side. Cranial nerve IX exits the jugular foramen and curves in close proximity under the SP.

SP has two embryological parts as tympanohyal in proximal and stylohyal distally. Tympanohyal part ossifies before birth, whereas that for stylohyal part occurs after birth. Styloid process, stylohyoid ligament and lesser cornu of the hyoid bone are derived from the cartilaginous element of the second branchial arch (Reichert's cartilage) and these three structures altogether constitute the 'stylohyoid chain' or 'stylohyoid complex' ${ }^{1}$. Normally the SP is about $2.5 \mathrm{~cm}$ in length, when it exceeds $3 \mathrm{~cm}$ it is said to be elongated and often found in $4 \%$ individual ${ }^{2,3,4}$

\section{Disscusion :}

During the routine dissection of an old aged male body bilaterally ossified stylohyoid chain was found. The chain was made up of two osselets (short bone) in right side and three osselets in left side. In between the osselets diarthrodial-like joint was seen. Stylohyoid chain was extending from the base of the skull to the body of hyoid 


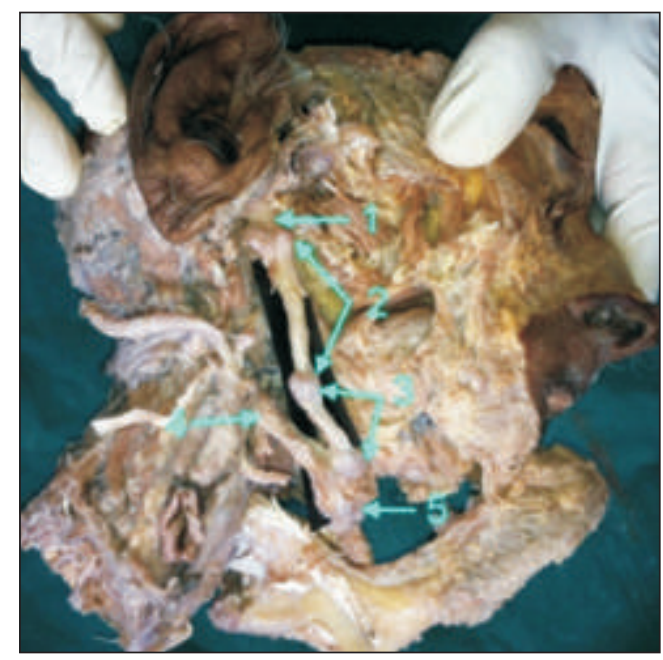

Right side stylohyoid chain

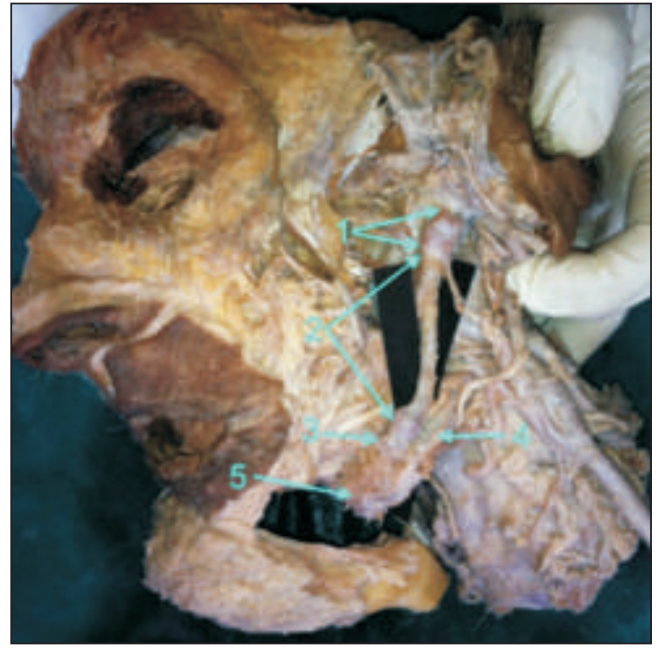

Left side stylohyoid chain

1-Tympanohyal 2-Stylohyal and ceratohyal 3- Hypohyal 4- Greater cornu of hyoid bone 5- Body of hyoid bone.

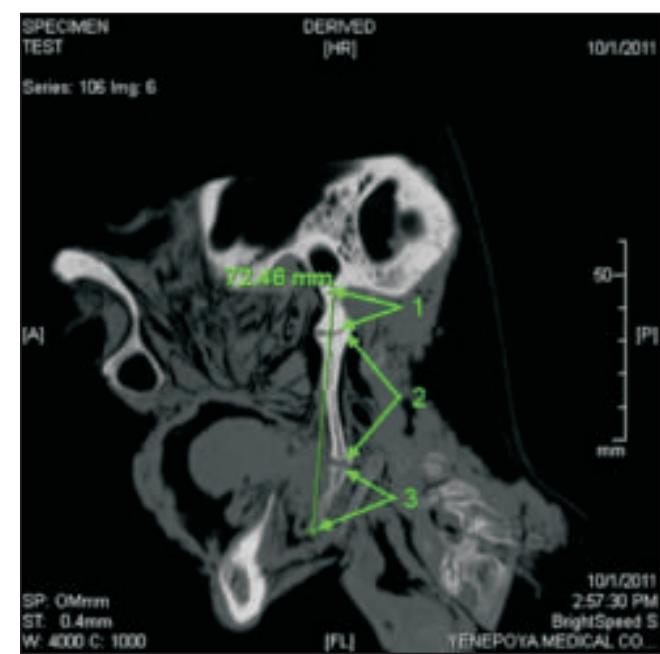

Right side stylohyoid chain

1-Tympanohyal 2-Stylohyal and ceratohyal 3- Hypohyal.

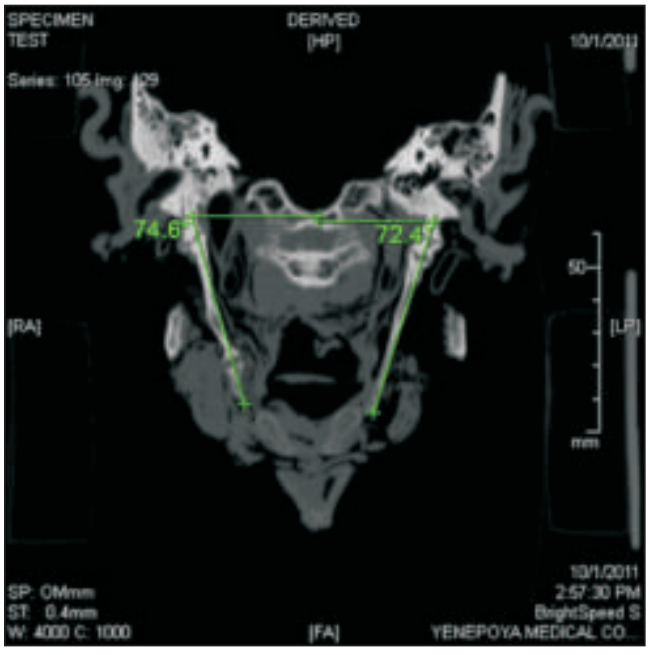

$2 \mathrm{D}$ image showing the angulations

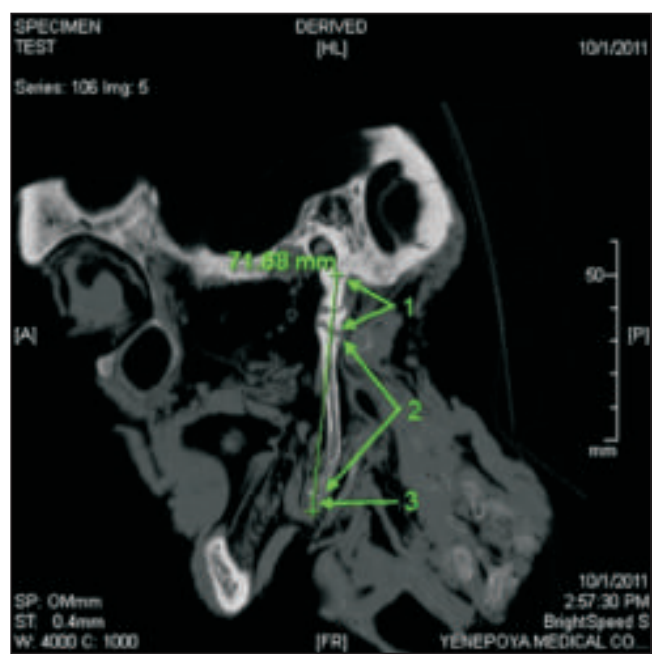

Left side stylohyoid chain

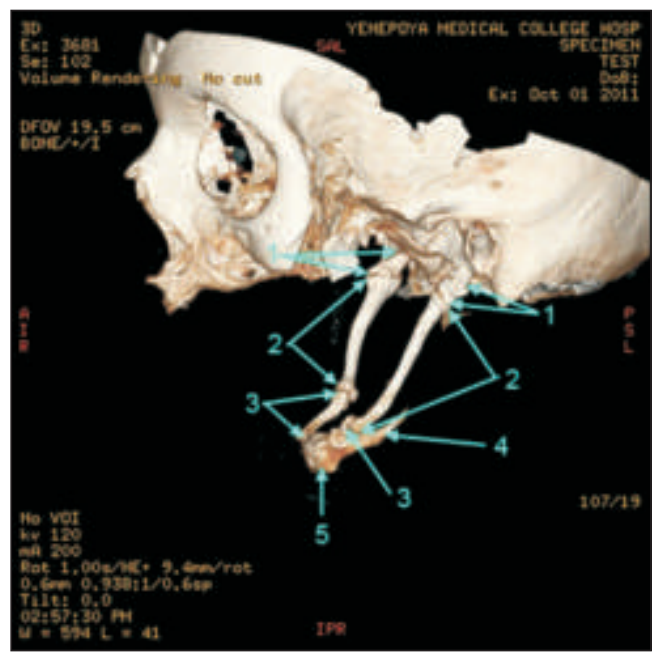

$3 \mathrm{D}$ image showing the parts

1-Tympanohyal 2-Stylohyal and ceratohyal 3-Hypohyal 4- Greater cornu of hyoid bone 5- Body of hyoid bone. 
bone. The specimen was scanned by CT scan and 3D image reconstruction was done. The length and medial angulations of the stylohyoid chain were measured. Length: Right side-72.46mm, Left side-71.68mm; Medial angulation: Right side-74.6 degrees, Left side-72.4 degrees.

After removing the nearby structures step by step we saw normal relations of stylohyoid chain. By studying the muscles attachment of different osselets of the chain we came to a conclusion thatstylohyal segment and ceratohyal segments are fused to form a single osselet, which articulates with tympanohyal part above and hypohyal part bellow. Hypohyal component not formed the lesser cornu but formed the distal osselet.

The components of the stylohyoid chain are derived embryologically from the second branchial arches in four distinct cartilaginous segments: tympanohyal, stylohyal, ceratohyal and hypohyal segments ${ }^{4}$.These segments are derived from Reichert's cartilages that ossify in 2 parts. The SP develops from the tympanohyal (first) and stylohyal (second) segments that usually fuse at puberty. The lesser cornu of the hyoid bone arises from the hypohyal (fourth) segment. Connecting these two structures, the stylohyoid ligament originates from the ceratohyal (third) segment. It is normally composed of dense fibrous connective tissue in adults but may retain its embryonic cartilage and thus have the potential for ossification. Excessive or abnormal ossification of stylohyoid chain components may result in several peculiarities including elongation of the SP and thickening of the hyoid bone. A solid stylohyoid chain results when the entire stylohyoid ligament ossifies, but many variations can occur including incomplete ossification, segmentation, pseudo-articulation and variations in thickness and angle. Ossified stylohyal ligament parts may merge or leave gaps in between.

A much rarer abnormality is a solid bar of bone connecting the hyoid bone with the base of the skull. An example of this was reported by Lipschuitz. This might possibly be due to ossification of embryonic cartilage, it apparently was not a senile change, as it was reported in a young person. Another form of abnormality is the presence of a styloid- hyoid chain of bones, such as found in the dog, horse, and many other animals. In such case the styloid process may be composed of two to five osselets, articulating by synchondrosis. Man is the only species which possessed a styloid process firmly ossified to the base of the skull. It is absent in monkeys.

Group of symptoms produced by elongated styloid process include foreign body sensation in the neck, hyper salivation, dysphagia as described by Eagle in 1937 as 'Eagle's syndrome ${ }^{15}$.The resultant abnormal stylohyoid chain may then compress or irritate nearby structures and is believed to be responsible for the stylohyoid syndromes ${ }^{6}$ .M any different names have been coined to describe the presence of head and neck symptoms associated with an elongated stylohyoid chain, including "Eagle's Syndrome", "Elongated Styloid Process Syndrome", "Carotid Artery Syndrome", "Styloid Process Neuralgia", "Stylalgia", "Stylohyoid Syndrome" and "Pseudohyoid Syndrome" "7,8,9.

\section{Conclusion :}

In this case we observed the stylohyoid chain, which may be due to ossification of the embryonic cartilage( Reichert's cartilages) in three segments in left side and four segments in right side. And also in between osselets there is a diarthrodial-like joint which allows the movement of hyoid bone during deglutition.

\section{Acknowledgements:}

The authors are grateful to Yenepoya University for permission to carry out this study.

\section{References:}

1. Williams PL. et al. Gray's Anatomy in: Giorgio Gabella.38 ${ }^{\text {th }}$ Edition. Churchill livingstone, 1995, pp. 592-593,561-562, 284.

2. Eagle's syndrome and the Trauma patient. Funct Ortho. 1997; 2264 916-925.

3. Feldman VB. Eagle's syndrome: a case of symptomatic calcification of the stylohyoid ligaments. J Can Chiropr Assoc. 2003; 47(1): 21-27.

4. Eagle WW. The symptoms, diagnosis and treatment of the elongated styloid process. Am Surg. 1962; 28: 1-5

5. Eagle WW. Elongated styloid process. Arch Otolaryngol. 1937; 25: 584-587.

6. Winkler S, Sammartino FJ, M onari JH. Stylohyoid Syndrome. Oral Surg Oral Med Oral Pathol. 1981; 51: 215-7.

7. Eagle WW. Elongated styloid process: report of two cases. Arch Otolaryngol.1937; 25: 584-7.

8. Gossman JR Jr, Tarsitano JJ. The styloid-stylohyoid syndrome. J Oral Surg. 1977; 35: 555-60.

9. Mukherjee P, Palit S, Tapadar A, Roy H., Asymmetrical bilateral ossification of stylohyoid chains - a case report with embryological review. International Journal of Anatomical Variations 2011; 4: 134-138. 